\title{
CARIOUS AND REMINERALISED ENAMEL; SYNCHROTRON X-RAY DIFFRACTION TEXTURE MAPPING
}

1. BDS, MSC (Oral Biology) Assistant Professor Islam Dental College Sialkot.

2. BDS, MSC (Oral Pathology) Assistant Professor Islam Dental College Sialkot.

3. BDS, FCPS (Operative Dentistry) Assistant Professor Islam Dental College Sialkot.

4. BDS, MSC (Operative Dentistry) Assistant Professor Isra Dental College Hyderabad.

5. Pharm-D, Ph.D

Lecturer

Faculty of Pharmacy,

University of Sindh, Jamshoro.

6. B-Pharm, Ph, D

Associate Professor

Faculty of Pharmacy,

University of Sindh, Jamshoro.

Correspondence Address:

Dr. Sana Zafar

Assistant Professor

BDS, MSC (Oral Biology)

Islam Dental College Sialkot.

danish_bds@yahoo.com

Article received on:

25/02/2017

Accepted for publication:

$15 / 05 / 2017$

Received after proof reading: 03/07/2017
Sana Zafar', Danish Javaid², Hanna Abdul Majeed ${ }^{3}$, Khurram Anwar', Mudassar Iqbal Arain ${ }^{5}$, Muhammad Ali Ghoto ${ }^{6}$

ABSTRACT... Objectives: The main purpose of this study was to determine from where the demineralisation of crystals occur in enamel during carious process and where the new crystals will form during remineralization. Material and Methods: Synchrotron x-ray diffraction technique was used to determine the crystal orientation and texture of enamel for a Period: of 02 years during 2010 and 2011. It was done on extracted teeth. 10 sides of a single tooth were analysed and a total of 100 teeth were examined. Sample analysis was done using composite imaging and powder diffraction using Fit 2D software. Settings: The study was done at Queen Mary University of London, Miles End Campus England. Sections of a tooth were taken having 1) healthy control enamel 2) natural carious enamel 3) artificially demineralized enamel and 4) artificially remineralized enamel. Synchrotron x-ray diffraction was carried out and two dimensional images of the areas of the tooth were collected. A $20 \mu \mathrm{mx} 20 \mu \mathrm{m}$ beam spot was used to collect images at high resolution in order to see the changes that occur in a small region of interest. First the Fit2D software was used for powder diffraction data processing and later Origin software was used obtain the texture values of the above mentioned areas. Results: Comparison of the side and cuspal both for the control and carious enamel showed that the carious enamel has a better texture than the control enamel. Also a repetition of the pattern after every 60 to 100 um was seen in the cuspal enamel both in the control and carious enamel respectively. Conclusion: The natural carious enamel showed a better texture and more orientation from the healthy control enamel. This indicates that the continuous processes of demineralization and remineralization which occur in enamel during caries may have some effects in increasing the texture of enamel since the mineral left in the carious enamel has a higher degree of crystallite orientation than the control enamel.

Key words: $\quad$ Enamel, Synchrotron X-ray, Diffraction and Remineralised.

Article Citation: Zafar S, Javaid D, Majeed HA, Anwar K, Arain MI, Ghoto MA. Carious and remineralised enamel; synchrotron x-ray diffraction texture mapping. Professional Med J 2017;24(7):1036-1043. DOI: 10.17957/TPMJ/17.3904

\section{INTRODUCTION}

Enamel an epithelial origin structure is the outer most covering of the tooth which protects the underlying structures from any physical and chemical damages. The cells, ameloblast, which produce the enamel, are lost when it is the time for tooth to erupt so that the enamel becomes an acellular structure which cannot regenerate. Therefore in order to overcome this phenomenon it has acquired a very complex structural organization having a higher mineral content as it matures. This shows the life cycle of ameloblasts and the physio-chemical properties of mineral proteins which results in the formation of thin and long enamel hydroxyapatite crystals. These properties differentiate the enamel from all the other tissues. The thickness of enamel varies in different areas of the tooth as the cusp has a thickness of approximately $2.5 \mathrm{~mm}$ and is at a minimum at the cervical areas. Therefore of this there is variation in the exact colour of enamel from being light yellow to greyish-white and also enamel is translucent so the underlying yellow dentine can be seen through the thin sections. Mature enamel consists of around $96 \%$ mineral which are mainly hydroxyapatite crystals and around $3 \%$ of organic matrix and $1 \%$ water.

Different ions like fluoride, strontium, magnesium and lead may get incorporated in the hydroxyapatite crystals during development. ${ }^{1}$ Some ions may replace the normal ions present 
in the enamel for example carbonate ions can replace the phosphate or carboxyl ions causing a stabilizing effect on the other hand magnesium can replace the calcium ions causing a destabilizing effect on the hydroxyapatite crystal structure. The hydroxyapatite crystals are large approximately $70 \mathrm{~nm}$ in width and $25 \mathrm{~nm}$ in thickness extending along the full width of the enamel tissue. One group i.e. $\mathrm{OH}$ with $3 \mathrm{Ca}$ ions having more 3 ions of phosphate were attached than finally six calcium ions covers the phosphate ions forming a hexagon. ${ }^{2}$

Water in the enamel causes porosity in the tissue. Water lies around the crystals and also around the organic material but some of the water can become entrapped causing defects in the crystals. The organic matrix is around $90 \%$ non-amelogenins like tuftlin and enamelin with small amount of amelogenins. When the enamel is developing this ratio is reversed. ${ }^{2}$ Enamel is basically made up of enamel prisms which run from the dentin enamel junction to the surface. When seen in crosssection the prisms in the human enamel appear to be 'keyhole' or 'tennis-racket' shaped and are placed in alternate ways that the tail of one prism is situated between the head of other two prisms. The prism is mainly formed by hydroxyapatite crystals and inter-prismatic substance is present which surrounds the crystals and join the crystals together. There is a small limit between the prism and inter-prismatic enamel known as the prism membrane or sheath. The prism membrane is absent at the cervical area of enamel so the crystals present in the inter-prismatic substance are confluent with the ones that form the prisms. When seen in longitudinal sections the lateral part of the prism crystals are in continuation with the inter-prismatic substance, situated in the cervical area till the crystals are almost perpendicular to the prism. ${ }^{1}$ Remineralization process can occur in all kind of carious lesion but the deeper the lesion the harder it is to remineralize. ${ }^{3}$

The pathological and protective factors determine whether the lesion will progress, stay the same or reverse. ${ }^{4}$ The carious process can be stopped or reversed if the protective factors predominate. It can also be assumed that the salivary dysfunction, cariogenic bacteria and the amount of fermentable carbohydrates taken are the pathological factors. Once the cariogenic bacteria make colonies on the tooth it is difficult to manage them. Components in saliva for example calcium, phosphate, fluoride proteins from the pellicle and proteins maintain the super saturation of minerals in the pellicle and saliva.

Anti-bacterial substances may be present in the saliva or provided externally for example from chlorhexidine. Remineralization process may occur in the following situations: In physiological condition: when the post-eruptive maturation is happening. Here the partially dissolved crystals get stored but where exactly the mineral deposition occurs is still unknown. It may restoration of the prism or the restoration of the inter-prismatic enamel. ${ }^{5-8}$ In pathological condition: where the demineralised areas are remineralized which maybe the expanding of inter-crystalline spaces or maybe the remineralization of the outer most layer of the carious ename. ${ }^{9-11}$

A synchrotron is a scientific research facility which produces an intense beam of x-rays, infrared and ultraviolet light which is named as synchrotron light. These are all a sections which can be detected by the human eye. Human eye can detect a range of visible light frequencies but there are a few electromagnetic frequencies that are not detected by it like the radio-waves, microwaves, UV (ultraviolet) light, x-ray and gamma rays.The synchrotron technique has the following advantages: Small samples can be taken, Sub-micron spatial resolution. Unlike a laboratory source, the x-rays energies can be tuned allowing them to enter deep down in the materials, A much higher intensity of the x-rays compared to a lab source, therefore, Reduced measurement time.

The objective of current study was to determine the effects of artificial and carious demineralization and remineralization on the crystalline structure orientation and surface defects of teeth while comparing it with that of normal enamel and to 
use synchrotron x-ray diffraction data analysis to provide novel insights into the enamel crystallites orientation during caries.

\section{METHODOLOGY}

\section{Sample Preparation}

Four different types of enamel were studied i.e. Lower molar tooth samples were collected from the patients who were undergoing treatments which required extractions from the institute of dentistry, Queen Mary university of London for a period of 02 years during 2010 and 2010. It was done on extracted teeth. 10 sides of a single tooth were analyzed and a total of 100 teeth were examined. Sample analysis was done using composite imaging and powder diffraction using Fit 2D software. The study was approved from ethical committee of concerned institute. Four different types of enamel were to be studied: Healthy control enamel, Natural carious enamel, artificially demineralised enamel, artificially remineralised enamel.

Artificial lesions were made exposing different tooth samples to acetic acid and later exposing half of them to remineralization solution (made of $\mathrm{CaCl}_{2}, \mathrm{KH}_{2} \mathrm{PO}_{4}, \mathrm{NaF}, \mathrm{NaCl}$ ) so as to mimic oral cavity environment but to study the texture of control and natural carious enamel the tooth which was studied in this thesis was not exposed to any solution. A diamond bur cutter was use $d$ to section the sample at the thickness of $500 \mu \mathrm{m}$ and samples were kept hydrated at all time as the tooth is naturally covered by saliva all the time.

In the synchrotron device the electron gun generates electrons which pass through three different accelerators called the linear accelerator or Linac, the booster synchrotron and finally through the large storage ring. The storage ring is a polygon which has straight sections which are angled together with the bending magnets. These magnets called the dipole magnets direct the electron through the storage ring and as these electrons comes in contact with magnets they lose energy in the form of light which is the synchrotron light stored in the beam lines. Each beam line is made in such a way that it carries out its own function. The Optics hutch is the first beam line where the light is filtered and focused. After that there is an experimental hutch, for the experiments to be carried out after that there is a control cabin where the scientist control the experiments and collect the data and results are produced as shown in the Figure-1.

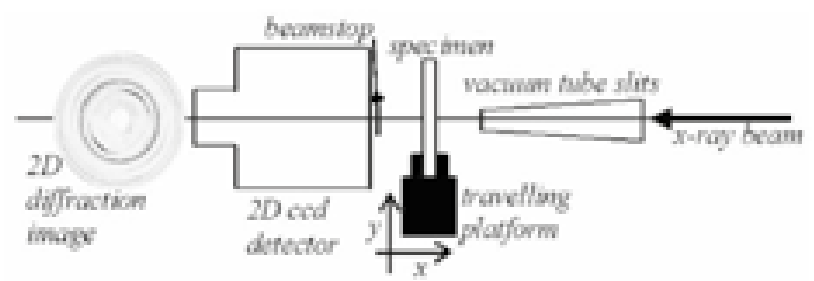

Figure-1 $1^{12} \mathrm{X}$-ray beam first passes through the vacuum tube slits into the specimen which is placed on a travelling platform into the 2D ccd detector and 2D diffraction image is produced.

The x-ray diffraction can be explained with the help of Bragg's Law which explains that the lattice parameter causes the reflection of X-rays which arise from the diffraction beams and if this beam is incident on the lattice plane with a space of $d$, then for an angle of incident $\theta$, the scattered $x$-rays emphasize in such a way that they can be measured to be reflected off the lattice planes. Difference in the path between the beams is reflected from successive planes which is $2 d \sin \theta$ being the constructive interference which must be an integral number of wavelengths. Hence $2 d$ sin $\theta=n \lambda$. The samples were to placed perpendicular to the beam line so they were mounted on a travelling platform. 2D x-ray diffraction patterns were collected on the XMaS beam line which is situated at the European Synchrotron Radiation Facility (ESRF), Grenoble.

Healthy control and carious enamel regions were identified within the sample tooth section to lower the effects of natural variability, by using the cross hairs on telescope of each sample. A beam spot of $20 \mu \mathrm{m} \times 20 \mu \mathrm{m}$ was used and the wavelengths of 0.82 Angstroms with the sample detector distance set at $180 \mathrm{~mm}$. High resolution images were collected by the MAR CCD detector so that changes across small area of interest can be detected. Each box of control and natural 
caries had a number of files from which were each interpreted as a separate file. To analyze the texture of control and natural carious regions Chiplots were created. Chiplot is an ascii file of two columns of data $X$ (Azimuthal angle value from 0 to 360) and $Y$ (intensity) using the same Fit2D software. It had two steps: Sample to detecto :f new materials so that ambiguities in structural determination are removed as in this the reflection positions are well characterized and do not overlap. In the Fit2D the commands "Calibrant_" and "_Lanthanum Hexaboride_" were carried out and values for the sample to detector distance and wavelength were entered which were $180 \mathrm{~mm}$ and 0.82 Angstroms respectively.

For Texture analysis Intensity vs. Azimuth curves were exported

After the above mentioned procedure was carried out the beam center was determined and circles were coordinated. When an ' $X$ ' appeared in the center of the image as shown the figure 2.2 it was then integrated. After that the azimuth angle was determined from 0 to 360 degrees and inner and outer radius was marked around the 002 reflection of enamel (Figure-3.8). The horizontal and vertical pixels sizes were set at 80 microns and the image was then integrated. Fit 2d displayed 1D intensity vs. azimuth plot which was then saved as Chiplot and uploaded in the origin software for texture analysis.

\section{Synchrotron X-ray Diffraction Data Analysis: Origin software}

After the powder diffraction was carried out the files were imported into the origin software (taken from: www.originlab.com) to analyze the 2 peaks of enamel and see the texture of enamel. This was carried out by plotting the two peaks of enamel at the intensity vs. azimuth angle graph around the $360^{\circ}$ Debye ring of the 002 reflection of enamel. These peaks were then fitted to the Gaussians peak shape plus background to see the orientation of the crystallites by shape quantification. The graph was plotted by taking the distance from the surface vs. the $\mathrm{w} 1$ and $\mathrm{w} 1$ error of all the files. Control 1, natural carious region 1 and 7 files were studied in the horizontal direction and control 2 and natural caries 6 files were studies in vertical directions.

\section{RESULTS}

The tooth was cut into sections of $500 \mu \mathrm{m}$ thickness and the control and the caries were compared for texture analysis. Control 1 was compared to natural caries 1 and 7 as they are the areas which are present on the side of the tooth and are closest spatially while control 2 was compared to natural caries 6 as both the regions lay on the cusp of the tooth as shown in the Figure-2.

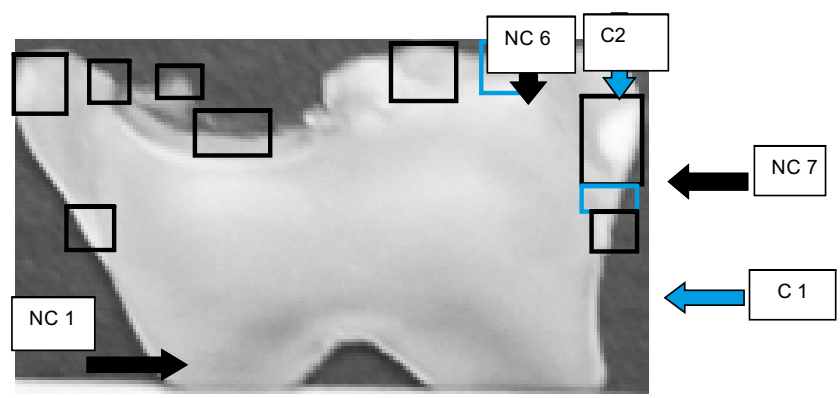

Figure-2. Showing the different areas of control and carious enamel that were identified at the Grenoble (ESRF).

When the files were analyzed in the Fit2D software, a composite image was formed for each area so as to differentiate between the air, enamel and dentine. The composite of air does not show any Bragg's peak while enamel has two peaks which show it is anisotropic and dentine has an isotropic feature. After that Intensity versus Azimuthal analysis of the 002 Bragg reflections was carried out and two pronounced peaks of enamel were seen in the Fit2D software. Each file in the control 1 and natural caries 1 and 7 was interpreted and it was seen in general that the control 1 peak was broader and of lower intensity as shown in Figure-3 than the caries peak 1 and 7 as shown in the Figures- 4 and 4.5 respectively when seen in intensity vs. azimuthal angle graph. The difference in the control and carious peaks is due to difference in intensity of the two peaks as the tilt of the peaks may be out of plane but as caries cause destruction of enamel minerals the difference in the peaks can also be because of this destruction. 


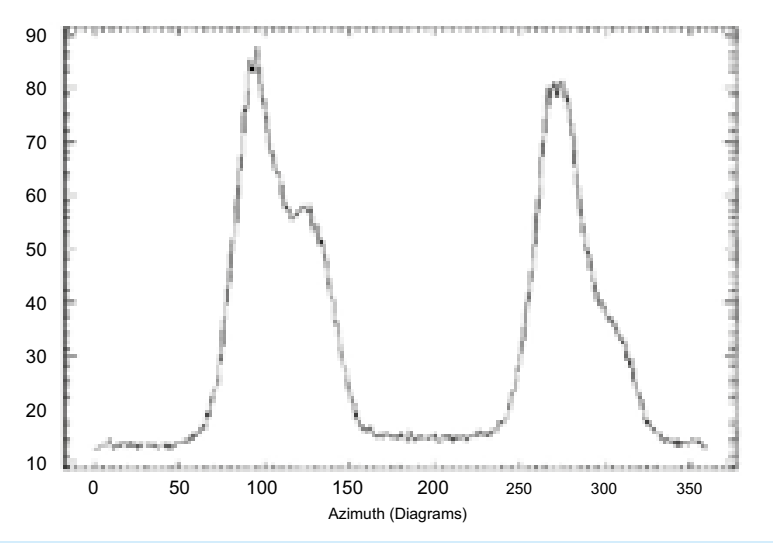

Figure-3. Intensity vs. Azimuth graph for control 1 enamel.

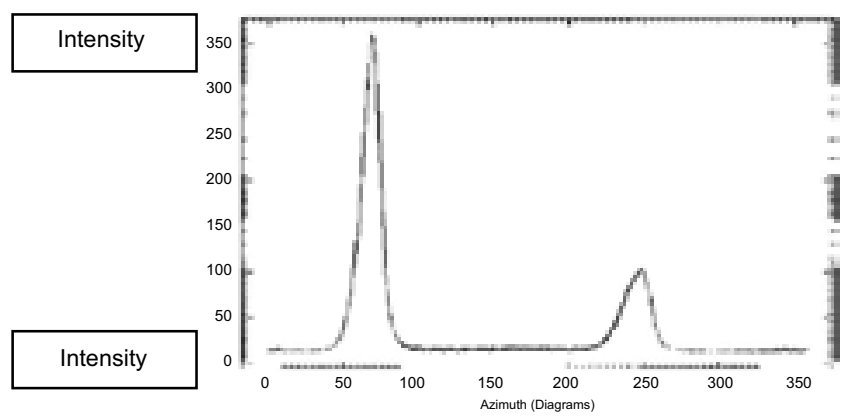

Figure-4. Intensity vs. Azimuth graph for caries 1 region.

Similar patterns to Figure-3 and 4 were shown for the control 2 and natural caries 6 regions which were present on the cusp of the tooth. Compares azimuth angles vs. the FWHM (full width half maximum) of a typical pattern from carious enamel, and healthy control enamel. This shows that there is a higher degree of texture in carious peak as compared to the control peak as the higher the values the lower the texture is and the lower the values the higher the texture and hence caries showing more texture. These results were then saved to be later viewed in the origin as Chiplot files. Graph between the distance and FWHM for texture analysis of natural caries 1 area showing that the caries have a higher texture. There was quite a difference in texture of control 1 , caries 1 and 7 region to further see if the same difference was in control 2 and region 6 same procedures were done and their results. Further the control 1 and carious region 1 and 7 graphs were compared as shown in Figure-4.14 in the origin software to compare the texture in all the of the above mentioned regions as they all were present on the side of the tooth. Showing the comparison between the control 1 and natural caries 1 and 7 and it can be seen that the values for the control 1 are higher than the caries. The same comparison was done between the control 2 and natural caries 6 region but the files here were interpreted in the vertical axis. This was later further researched and seen that the control 2 had the same pattern being repeated after 60 microns and caries had the similar pattern repeated by 100 microns. Same repetation of pattern was seen in caries region 6 but it was after every 100 microns. The data analyzed for this thesis was limited to carious/unaffected enamel. However these results to see the texture of control enamel and natural caries can be compared with the artificially demineralized and remineralized enamel

\section{Discussion}

Mineral loss from the tooth enamel in natural caries is an unavoidable phenomenon and this process of demineralization has been previously studied by many researchers. Synchrotron X-Ray diffraction is a relatively new technique that helps to study the loss of mineral from the exact site and also where the new mineral will be laid down. The data in the graph shown in Figure-3 indicates that the natural caries have a higher texture than the control enamel as the higher the values for FWHM the lower the texture. It can also be seen that as the distance increases the control 1 there is a sudden increase in texture between 100$200 \mu \mathrm{m}$ while the natural caries 1 have a sudden decrease in texture at the same point. Also as the distance increases the texture of natural caries 1 decreases indicating that the mineral loss is more near the dentine enamel junction as compared to the surface of enamel. Natural caries 7 show an almost straight line showing that the mineral loss had been constant and it had been from such places as to give a better texture to that area. The texture of the carious lesion 1 and 7 may seem to be improving because of the constant remineralization that occurs naturally in the oral cavity or maybe the mineral loss has occurred but the orientation of the crystals was not disturbed during this process. This difference in the texture 
was also seen $b^{y 13}$ as the results from SAXS (small angle x-ray diffraction) showed that the control enamel shows a weak equatorial scatter at $100 \mu \mathrm{m}$ from the surface when compared to the carious enamel and this high carious scatter was enhanced in the demineralized zone at $100 \mu \mathrm{m}$ from the surface. This difference in the scatter may be because of the difference in the hydroxyapatite crystals and the amount of non-mineral substance i.e. water and carbonate between them. Electron microscopy have shown that the demineralization process cause a decrease in the hydroxyapatite crystals and an increase in voids ${ }^{13}$ but the loss of mineral can be in such a way as to enhance the texture of the remaining crystals. Tissue destruction in caries has no preferential destruction pattern of prismatic and inter-prismatic enamel. It has been postulated by Sharpe (1965, 1967 ) that the dissolution of crystals occur only in those areas where the crystals are present endon and because the different crystal orientation of the head and tail area of prism and between inter prismatic and intra prismatic enamel different results can be seen with different techniques. ${ }^{14}$

X-ray diffraction done on the cusp of a permanent tooth in the mesio-distal direction showed that the hydroxyapatite crystals have a higher degree of preferred orientation as they lie almost parallel to the prism in this direction but there is a change in this pattern if moved away from the cusp to the sides. It was also seen that lateral enamel show variations from cusp to cervical margin as there is tendency for the hydroxyapatite crystals to deviate more from the prism axis. ${ }^{15}$ When the comparison between the control 2 and natural caries 6 (both areas on the cusp of tooth) was done the same results as before were seen i.e. the carious enamel had a better texture than the control enamel. Therefore there dissolution of minerals in the carious enamel must be in such a way to increase the texture of the remaining crystals. Also both the control 2 and natural caries 6 the enamel seems to follow a pattern which was being repeated after every 100 to $60 \mu \mathrm{m}$ respectively. This change of distance of the same pattern being repeated can be due to change in the biological structure and location as the carious is a bit mesial to the control enamel which is right on the top of the cusp of the tooth and it is known that the crystals deviate gradually from the cusp to cervical margins. This repetition of the same pattern shows there must be some structure that is present at that particular distance and studies done previous have shown that it could be the characteristics of lines of Retzius. It has been seen through SEM ${ }^{16-19}$ that they enamel rods have a wavy pattern and that they run obliquely and more and more undulated towards the enamel surface so enamel mantle has to fill in the space between them. They run to around 40um to $100 u m$ from dentin enamel junction but some of them run through the full length of enamel. It is also know that these lines of Retzius show a resting period between the deposition and secretion of enamel and these areas are represented as hypominerlisation ${ }^{20}$ and hence there was a depression seen in the graph after every 60 to 100 um. Distance between the lines of Retzius can be between 4 and $150 \mu \mathrm{m}$ it decreases from the center to periphery of the tooth. Change in the distance of the repetition of the structure can be related to the "two-fiber axes" which are because of the orientation of the crystals. The crystals are arranged in two distinct ways in human enamel which is because of the fact that two groups of apatite are present in enamel. One group of the prism deviates at 5 degree whiles other deviates at 40 degree. At first Poole and Brooks (1961) concluded that this problem maybe an artifact which may be because of the $\mathrm{x}$-ray beam may have inclusion of two different enamel layers but later studies done by Glas $^{21}$ concluded that the crystals on c-axes combine together along one or two directions.

\section{CONCLUSION}

Synchrotron X-ray diffraction showed that in general both on the side and cuspal enamel the natural caries have a higher texture than the control enamel indicating the fact that there must be destruction/dissolution of such structures that helps to improve the texture of the carious enamel or it could be that when mineral loss occurs in caries the orientation of crystals is not disturbed hence improving the texture. Still further studies 
are required to see what exactly occurs in the carious enamel as to improve its texture when compared to normal enamel.

\section{Clinical Significance}

The early diagnosis of caries formation and the process of deminerlization and reminerlization will help us to promote the reminerlization of carious tooth and prevent it from further destruction. Try and develop improved treatment for the remineralization of dental caries so that less money is spent on dental procedures.

Copyright(c) 15 May, 2017.

\section{REFERENCES}

1. Mihaela, C. MIHU., Dudea, D., Melincovivi, C., Bosca, B. 'Tooth enamel, the result of the relationship between matrix proteins and hydroxyapatite crystals'. 2008; Vol 23, No.3-4 pg 68-72.

2. Berkovitz, B.K.B., Moxham, B.J., Linden, R.W.A., Sloan, A.J. 'Master dentistry' 2011; Volume3, chapter 12.p 142-147.

3. Ten Cate, J.M., Featherstone, J.D. 'Mechanistic aspects of the interactions between fluoride and dental enamel'. CRC Crit Rev OralBiol Med 1991;2:283296.

4. Featherstone, J.D. The science and practice of caries prevention. JAm Dent Assoc 2000;'131:887-899.

5. Kawasaki, K., Ishikawa, T., Fearnhead, R.W., Mok, Y.C., Shimanoke, Y and Inoue, K. 'Quality variation in tooth enamel. In: Tooth Enamel IV'edsFearnhead R W and Suga S, pp. 1984;331-337, (Elsevier Science, Amsterdam).

6. Tohda, $\mathrm{H}$. 'Forming mechanism of subsurface carious lesions in enamel'. ShikwaGakuho 1981;81: 421-444.

7. Tanaka, N. 'High resolution electron microscopy of the crystalline structure in remineralized enamel'. ShikwaGakuho 1989;89: 1441-1478.

8. Tohda, H., Yanagisawa, T., Tanaka,N and Takuma, S. 'Growth and fusion of apatite crystals in the remineralized enamel'. J. Electron Microsc. 2011;39: 238-244.

9. Takuma.S. 'Demineralization and remineralization of tooth substances. An ultrastructural basis for caries prevention' J. Dent. Res. 1980;59: 2145-2156.
10. Yanagisawa, T. 'Crystalline structure of enamel in carious lesion'. J. Jpn. Dent. Ass. 1997;46: 1167-1176.

11. Yanagisawa, T., Takuma, S., Tohda, H., Fejerskov, O., and Fearnhead, R.W. 'Resolution electron microscopy of enamel crystals in cases of human dental fluorosis'. J. Electron Microsc. 2011;38: 442-448.

12. Al-jawad, M., Steuwer, A., Kilcoyne,S., Shore,R., Cywinski,R.,Wood,D. 2D mapping of texture and lattice parameters of dental enamel'. Biomaterials 2007;28.2908-2914.

13. Yagi,N., N., Ohta, T., Matsuo, Tanaka, T., Y., Terada, H., Kamasaka,K., to-o,K., Kometani,T., Kuriki, T. 'Evaluation of enamel crystallites in subsurface lesion by microbeam x-ray diffraction'. J.Synchrotron Rad.2011;16, 398-404.

14. Johnson, N. W. (1967). 'Some aspects of the ultrastructure of early human enamel caries seen with the electron microscope'. Archs oral Biol. Vol.12, pp. $1967 ; 1505-1521$.

15. Poole, D. F. G. and Brooks, A.W. 'The arrangement of crystallites in enamel prisms' Arch. Oral biol. 1961; Vol.5, pp.14-26.

16. Boyde, A. 'The structure and development of mammalian enamel'. PhD. thesis, University of London 1964.

17. Radlanski, R. J. 'Micromorphological features of human dental enamel'. In: Alt KW, R€osing FW, Teschler-Nicola $\mathbf{M}$, editors. Dental anthropology Fundamentals, limits, and perspectives.Wien/New York: Springer; p. 1998;129-45.

18. Radlanski, R. J., Seidl, W., Steding, G. 'Prism orientation in human dental enamel'. In: Moggi-Cecchi J, editor. Aspects of dental biology: palaeontology, anthropology, evolution. Florence: International Institute for the Study of Man (Angelo Pontecorboli); 1995. p. 33-49.

19. Radlanski, R.J., Renz, H., Willersinn, U., Cordis, C. A., Duschner, $H$. 'Outline and arrangement of enamel rods in human deciduous and permanent enamel'. 3D-reconstructions obtainedfrom CLSM and SEM images based on serial ground sections. Eur J Oral Sci; 2010;109:409-14.

20. Gustafson, G. 'Microanatomy and histochemistry of enamel'. New York: Academic Press 1967.

21. Glas, J.E., Nylen, M.U. (1965) 'A correlated electron microscopic and microradiographicstucy oh human enamel'. Arch oral boil.10.893-908. 


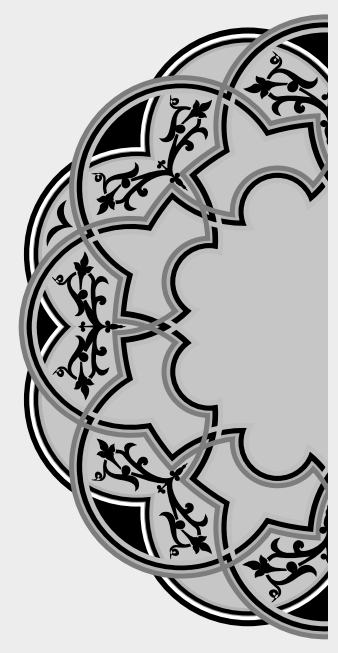

\title{
"If you can't explain it simply, you don't understand it well enough."
}

\author{
Albert Einstein
}

AUTHORSHIP AND CONTRIBUTION DECLARATION

\begin{tabular}{|c|c|c|c|}
\hline Sr. \# & Author-s Full Name & Contribution to the paper & Author $=\mathbf{s}$ Signature \\
\hline 1 & Dr. Sana Zafar & $\begin{array}{l}\text { Concept of study, } \\
\text { manuscript writing }\end{array}$ & 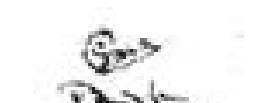 \\
\hline 3 & Dr. Hanna Abdul Majeed & COmposing of results & 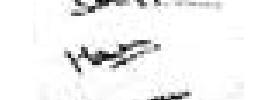 \\
\hline 4 & Dr. Khurram Anwar & Guide \& Writing & \\
\hline 5 & Dr. Mudassar lqbal Arain & Proof reading & \\
\hline 6 & Dr. Muhammad Ali Ghoto & Proof reading & \\
\hline
\end{tabular}

